\title{
Effective adsorptive removal of methylene blue from water by didodecyldimethylammonium bromide-modified brown clay
}

Muhammad Munir ${ }^{a}$, Muhammad Faizan Nazar ${ }^{a *}$, Muhammad Nadeem Zafar ${ }^{\text {** }}$, Muhammad Zubair $^{\mathrm{a}}$, Muhammad Ashfaq ${ }^{\mathrm{a}}$, Ahmad Hosseini-Bandegharaeib,c, Salah Ud-Din Khan ${ }^{\mathrm{d}}$, Ashfaq Ahmad $^{\mathrm{e}}$

aDepartment of Chemistry, University of Gujrat, Gujrat, 50700 Pakistan

bDepartment of Environmental Health Engineering, Faculty of Health, Sabzevar University of Medical Sciences, Sabzevar, Iran

'Department of Engineering, Kashmar Branch, Islamic Azad University, PO Box 161, Kashmar, Iran

dSustainable Energy Technologies Center, King Saud University, P.O. Box 800, Riyadh 11421, Saudi Arabia

eDepartment of Chemistry, King Saud University, P.O. Box 2425, Riyadh 11451, Saudi Arabia

\section{Supporting Information}




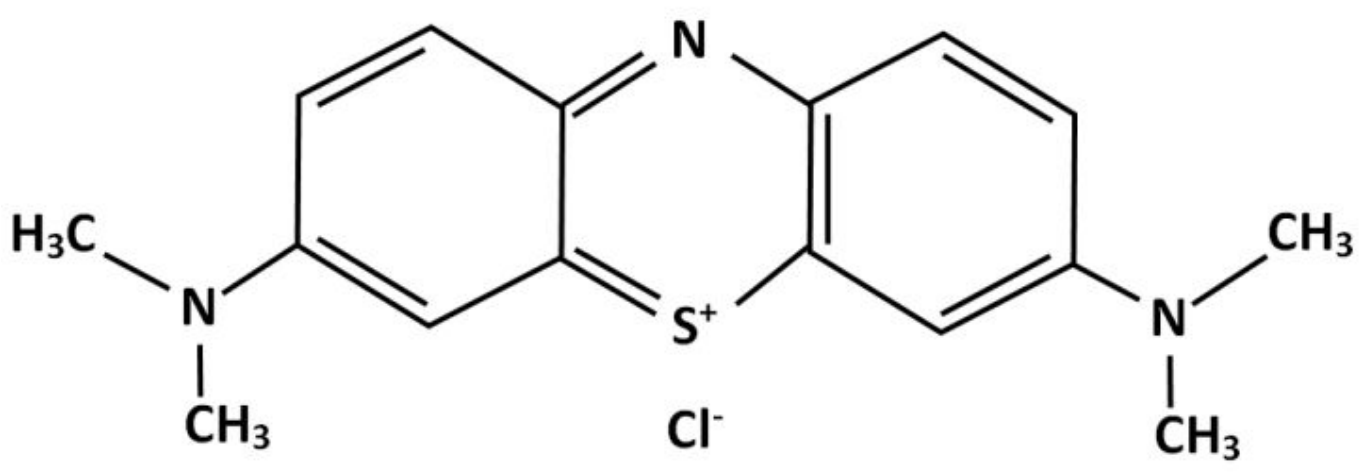

Figure S1. Molecular structure of methylene blue (MB) dye 


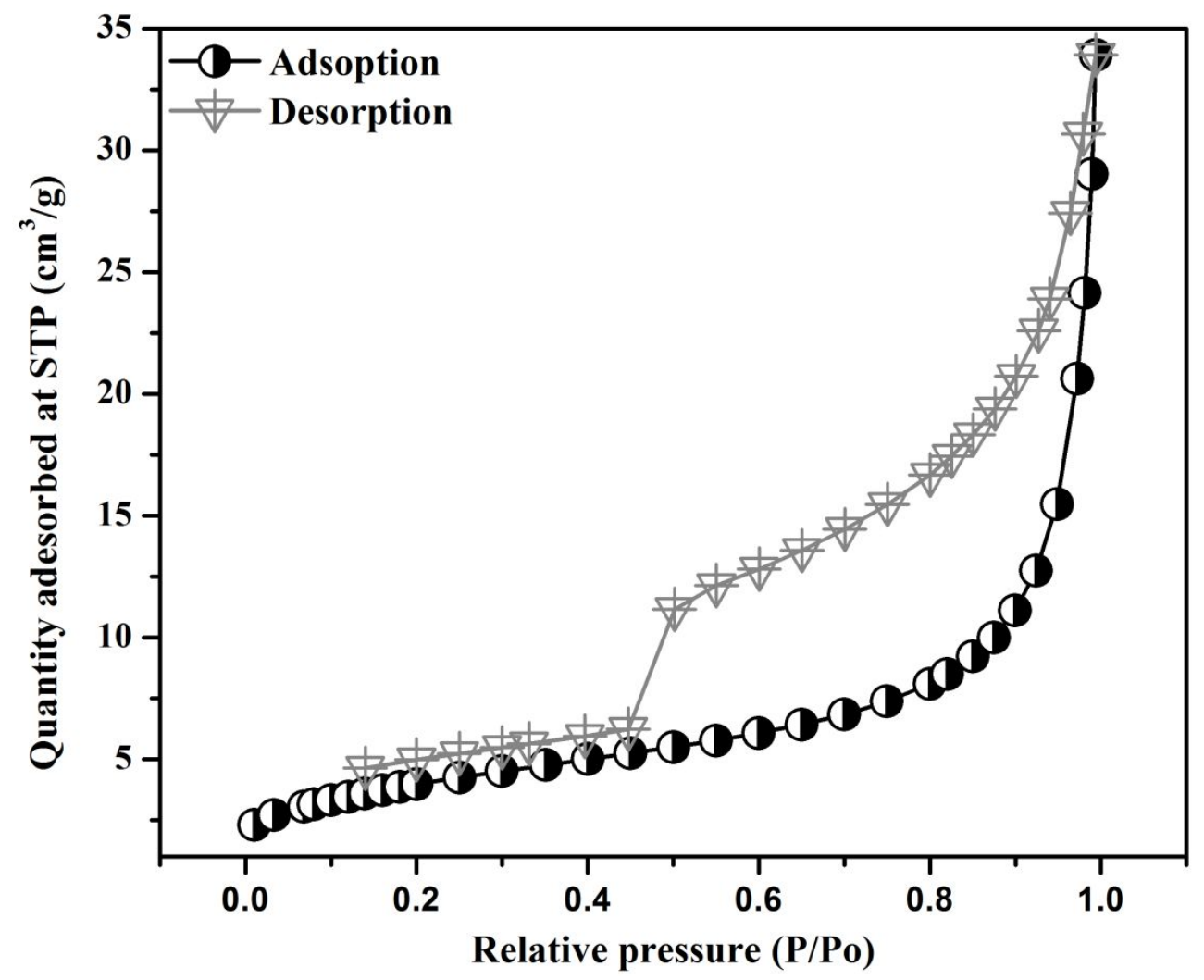

Figure S2. BET isotherm plot of BC sorbent 
Table S1. BET surface parameters of BC and DDAB-BC

\begin{tabular}{|c|c|}
\hline Parameters & Values \\
\hline \multicolumn{2}{|c|}{$\mathrm{BC}$} \\
\hline Surface area & $14.5884 \mathrm{~m}^{2} / \mathrm{g}$ \\
\hline Pore diameter & $10.16264 \mathrm{~nm}$ \\
\hline Pore volume & $0.031916 \mathrm{~cm}^{3} / \mathrm{g}$ \\
\hline \multicolumn{2}{|c|}{ DDAB-BC } \\
\hline Surface area & $124.6841 \mathrm{~m}^{2} / \mathrm{g}$ \\
\hline Pore diameter & $8.75102 \mathrm{~nm}$ \\
\hline Pore volume & $0.316780 \mathrm{~cm}^{3} / \mathrm{g}$ \\
\hline
\end{tabular}




\section{Table S2. Composition of BC}

\begin{tabular}{lc} 
Components & Composition (\%) \\
\hline $\mathrm{SiO}_{2}$ & $54-60$ \\
$\mathrm{Al}_{2} \mathrm{O}_{3}$ & $21-25$ \\
$\mathrm{Fe}_{2} \mathrm{O}_{3}$ & $8.9-9.3$ \\
$\mathrm{Cu}$ & $0.4-1.54$ \\
$\mathrm{MgO}$ & $2.23-2.65$ \\
Loss on ignition & 7.25 \\
Density & $1.23\left(\mathrm{~g} / \mathrm{cm}^{3}\right)$ \\
Moisture & $2.5 \%$ \\
Water solubility & $2.41 \%$ \\
$\mathrm{pH}$ & 7.1 \\
\hline
\end{tabular}




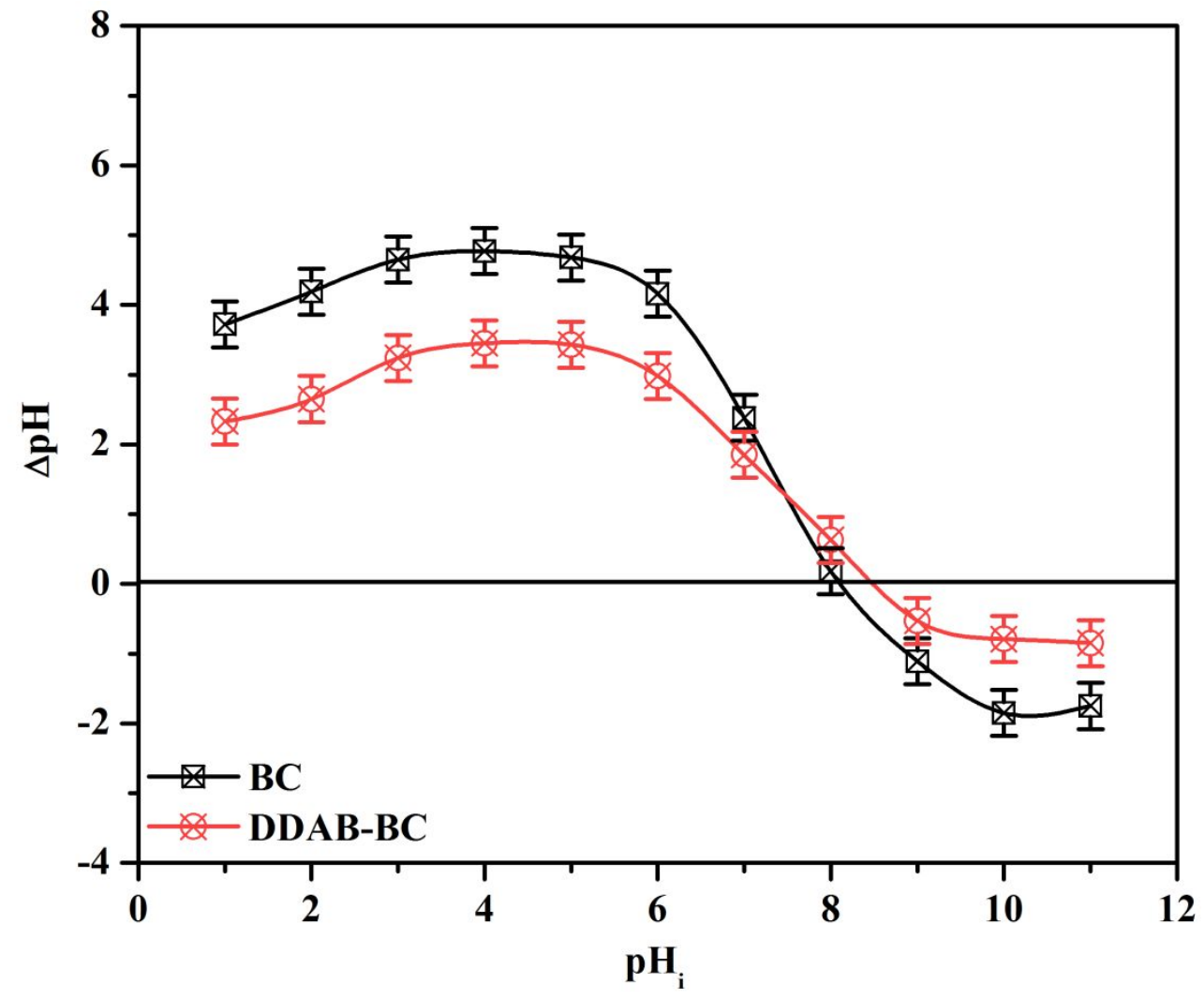

Figure S3. The point of zero charge $\left(\mathrm{pH}_{\mathrm{PZC}}\right)$ of $\mathrm{BC}$ and $\mathrm{DDAB}-\mathrm{BC}$ sorbents 


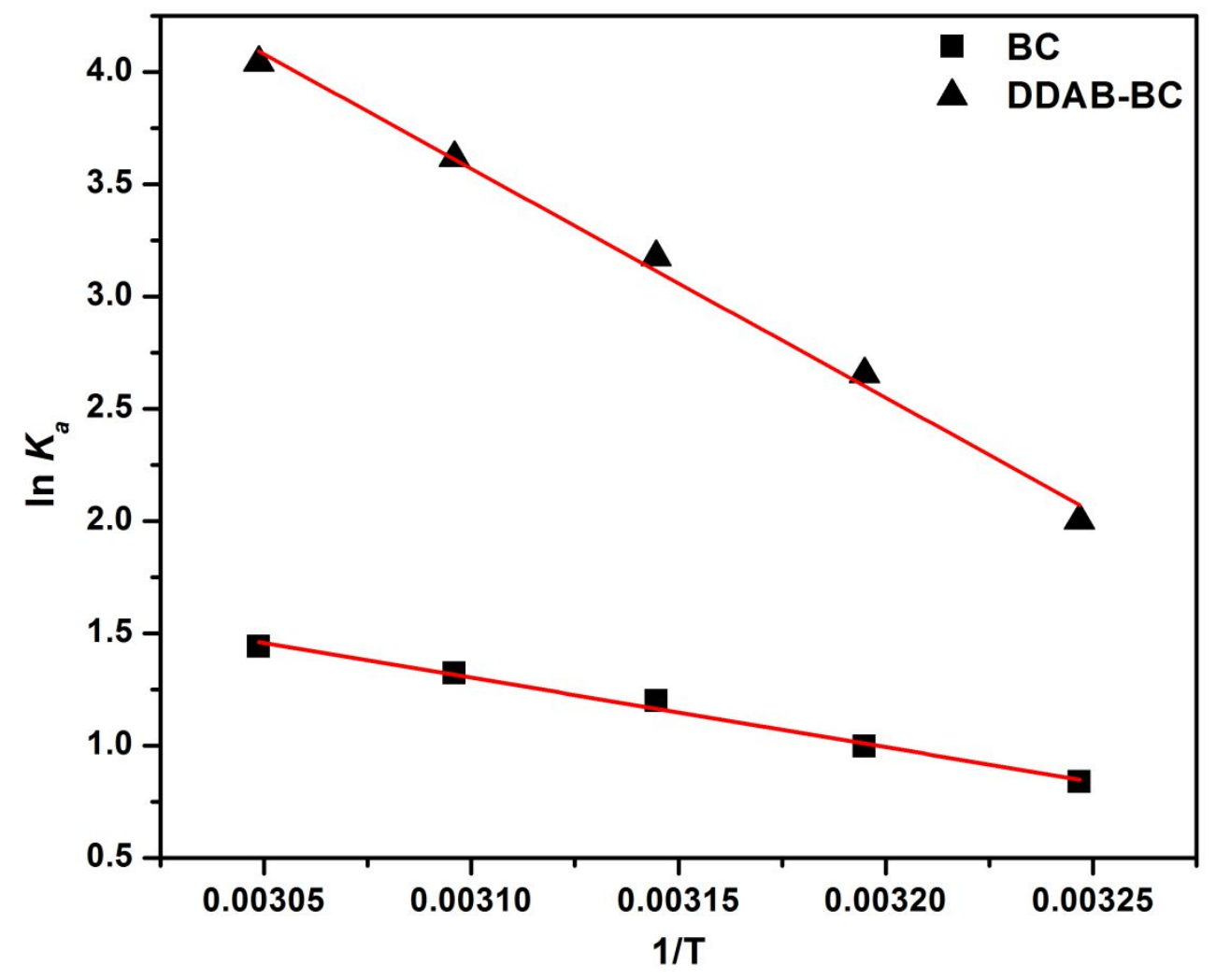

Figure S4. Plots of Ln $K_{a}$ vs. 1/T for MB sorption onto BC and DDAB-BC 